International Journal on Cybernetics \& Informatics (IJCI) Vol. 4, No. 5, October 2015

\title{
A COMPARISON OF VARIOUS TYPE OF SOFT COMPATIBLE MAPS AND COMMON FIXED POINT THEOREM - II
}

\author{
Sumit Mohinta and T. K. Samanta \\ Department of Mathematics, Sudhir Memorial Institute, Kolkata, West Bengal, India- \\ 700132. \\ Department of Mathematics, Uluberial College, Uluberia, Howrah, West Bengal, India- \\ 711315.
}

\begin{abstract}
In this article, first we generalize a few notions like $(\alpha)$ - soft compatible maps, $(\beta)$ - soft ompatible maps, soft compatible of type (I) and soft compatible of type (II ) maps in oft metric spaces and then we give an accounts for comparison of these soft compatible aps. Finally, we demonstrate the utility of these new concepts by proving common fixed point theorem for fore soft continuous self maps on a complete soft metric space.
\end{abstract}

\section{KEYWORDS:}

soft metric space, soft mapping, soft compatible maps, $(\alpha)$ - soft compatible maps, ( $\beta$ ) - soft compatible maps, soft compatible of type ( I ), soft compatible of type ( II ), common fixed point.

2010 Mathematics Subject Classification: 03E72, 08A72, 47H10, 54H25

\section{INTRODUCTION}

The soft set theory, initiated by Molodtsov [3] in 1999, is one of the branch of mathematics, which targets to explain phenomena and concepts like ambiguous, undefined, vague and imprecise meaning. Soft set theory provides a very general frame work with the involvement of parameters. Since soft set theory has a rich potential, applications of soft set theory in other disciplines and real life problems are progressing rapidly.

In recent time, researchers have contributed a lot towards soft theory . Maji. et al. [5, 6] introduced several operations on soft sets and applied it to decision making problems. A new definition of soft set parameterization reduction and a comparison of it with attribute reduction in rough set theory was given by Chen [2].

Then the idea of soft topological space was first given by M.Shabir and M.Naz [4] and mappings between soft sets were described by P.Majumdar and S.K.Samanta [7]. Later, many researches about soft topological spaces were studied in $[12,13,14]$. In these studies, the concept of soft 
International Journal on Cybernetics \& Informatics (IJCI) Vol. 4, No. 5, October 2015

point is expressed by different approaches. Then S. Das and S. K. Samanta [8] introduce the notion of soft metric space and investigated some basic properties of this space. Later on different kinds of fixed point theorem have been established in soft metric spaces.

In this article, a generalization of ( $\alpha$ ) - soft compatible maps, ( $\beta$ )- soft compatible maps, soft compatible of type ( I ) and soft compatible of type ( II ) maps are introduced in soft metric spaces and then we give an accounts for comparison of these soft compatible maps. Finally, we have proved a common fixed point theorem for fore soft continuous self maps on a complete soft metric space. In order to add validity and weight to the argument that our concept is viable and meaningful in the context of fixed point theory in soft metric spaces, we have presented a series of Propositions and Examples in support of pertinent results.

\section{PRELIMINARIES}

3.the intersection of any finite number of soft sets in $\tau$ belongs to $\tau$. The triplet $(X, \tau, E)$ is called a soft topological space over $X$. Definition 2.1. [3] Let $X$ be an initial universe set and $E$ be a set of parameters. A pair $(F, E)$ is called a soft set over $X$ if and only if $F$ is a mapping from $E$ into the set of all subsets of the set $X$, i.e., $F: E \rightarrow P(X)$, where $P(X)$ is the power set of $X$.

Definition 2.2. [8] A soft set $(P, E)$ over $X$ is said to be a soft point if there is exactly one $e \in E$, such that $P(e)=\{x\}$ for some $x \in X$ and $P\left(e^{\prime}\right)=\phi, e^{\prime} \in E-\{e\}$. It will be denoted by $\tilde{x}_{e}$.

Definition 2.3. [8, 10] Two soft point $\tilde{x}_{e}, \tilde{y}_{e^{\prime}}$ are said to be equal if $e=e^{\prime}$ and $P(e)=P\left(e^{\prime}\right)$ i.e., $x=y$. Thus $\tilde{x}_{e} \neq \tilde{y}_{e} e^{\prime} \Leftrightarrow x \neq y$ or $e \neq e^{\prime}$.

Definition 2.4. [9] Let $R$ be the set of real numbers, $\wp(R)$ be the collection of all nonempty bounded subsets of $R$ and $A$ be a set of parameters. Then a mapping $F: A \rightarrow \wp(R)$ is called a soft real set. It is denoted by $(F, A)$. If in particular $(F, A)$ is a singleton soft set, then identifying $(F, A)$ with the corresponding soft element, it will be called a soft real number.

Definition 2.5. [9] Let $(F, A)$ be a soft real set and $\overline{0}$ be the soft real set such that $\overline{0}(\lambda)=\{0\}, \forall \lambda \in A$. Then $(F, A)$ is said to be a 
International Journal on Cybernetics \& Informatics (IJCI) Vol. 4, No. 5, October 2015

(i ) positive soft real set if $\overline{0}<_{s}(F, A)$,

(ii ) negative soft real set if, $(F, A)_{<_{S}} \overline{0}$.

(ii ) non-negative soft real set if $F(\lambda)$ is a subset of the set of non-negative real numbers for each $\lambda \in A$.

(iv ) non-positive soft real set if $F(\lambda)$ is a subset of the set of non-positive real numbers for each $\lambda \in A$.

Definition 2.6. [11] Let $\tau$ be the collection of soft sets over $X$, then $\tau$ is said to be a soft topology on $X$ if

1. $\Phi, X \in \tau$.

2. the union of any number of soft sets in $\tau$ belongs to $\tau$.

Definition 2.7. [1] Let $(X, \tau, E)$ and $\left(Y, \tau^{\prime}, E\right)$ be two soft topological spaces, $f:(X, \tau, E) \rightarrow\left(Y, \tau^{\prime}, E\right)$ be a mapping. For each soft neighborhood $(H, E)$ of $\left(f(x)_{e}, E\right)$, if there exists a soft neighborhood $(F, E)$ of $\left(\tilde{x}_{e}, E\right)$ such that $f((F, E)) \subset(H, E)$, then $f$ is said to be soft continuous mapping at $\left(\tilde{x}_{e}, E\right)$.

If $f$ is soft continuous mapping for all $\left(\tilde{x}_{e}, E\right)$, then $f$ is called soft continuous mapping.

Let $\tilde{X}$ be the absolute soft set i.e., $F(e)=X, \forall e \in E$, where $(F, E)=\tilde{X}$ and $\operatorname{SP}(\tilde{X})$ be the collection of all soft points of $\tilde{X}$ and $\square(E)^{*}$ denote the set of all non-negative soft real numbers.

Definition 2.8. [8] A mapping $\tilde{d}: S P(\tilde{X}) \times S P(\tilde{X}) \rightarrow \square(E)^{*}$, is said to be a soft metric on the soft set $\tilde{X}$ if $\tilde{d}$ satisfies the following conditions:

$\left(M_{1}\right) \tilde{d}\left(\tilde{x}_{e_{1}}, \tilde{y}_{e_{2}}\right) \tilde{\geq} \overline{0} \quad \forall \tilde{x}_{e_{1}}, \tilde{y}_{e_{2}} \tilde{\in} \tilde{X}$

$\left(M_{2}\right) \tilde{d}\left(\tilde{x}_{e_{1}}, \tilde{y}_{e_{2}}\right)=\overline{0}$ if and only if $\tilde{x}_{e_{1}}=\tilde{y}_{e_{2}} ;$

$\left(M_{3}\right) \tilde{d}\left(\tilde{x}_{e_{1}}, \tilde{y}_{e_{2}}\right)=\tilde{d}\left(\tilde{y}_{e_{2}}, \tilde{x}_{e_{1}}\right) \quad \forall \tilde{x}_{e_{1}}, \tilde{y}_{e_{2}} \tilde{\in} \tilde{X}$

$\left(M_{4}\right)$ For all $\tilde{x}_{e_{1}}, \tilde{y}_{e_{2}}, \tilde{z}_{e_{3}} \tilde{\in} \tilde{X}, \tilde{d}\left(\tilde{x}_{e_{1}}, \tilde{z}_{e_{3}}\right) \tilde{\leq} \tilde{d}\left(\tilde{x}_{e_{1}}, \tilde{y}_{e_{2}}\right)+\tilde{d}\left(\tilde{y}_{e_{2}}, \tilde{z}_{e_{3}}\right)$ 
International Journal on Cybernetics \& Informatics (IJCI) Vol. 4, No. 5, October 2015

The soft set $\tilde{X}$ with a soft metric $\tilde{d}$ on $\tilde{X}$ is called a soft metric space and denoted by $(\tilde{X}, \tilde{d}, E)$.

Definition 2.9. [8] Let $(\tilde{X}, \tilde{d}, E)$ be a soft metric space and $(\tilde{\varepsilon})$ be a non-negative soft real number. $B\left(\tilde{x}_{e}, \tilde{\varepsilon}\right)=\left\{\tilde{y}_{e} \tilde{\in} \tilde{X}: \tilde{d}\left(\tilde{x}_{e}, \tilde{y}_{e^{\prime}}\right) \tilde{<} \tilde{\varepsilon}\right\} \subset S P(\tilde{X})$ is called the soft open ball with center at $\tilde{x}_{e}$ and radius $\tilde{\varepsilon}$ and $B\left(\tilde{x}_{e}, \tilde{\varepsilon}\right)=\left\{\tilde{y}_{e} \tilde{\in} \tilde{X}: \tilde{d}\left(\tilde{x}_{e}, \tilde{y}_{e^{\prime}}\right) \tilde{\leq} \tilde{\varepsilon}\right\} \subseteq S P(\tilde{X})$ is called the soft closed ball with center at $\tilde{x}_{e}$ and radius $\tilde{\varepsilon}$.

Definition 2.10. [8] Let $(\tilde{X}, \tilde{d}, E)$ be a soft metric space and $(F, E)$ be a non-null soft subset of $\tilde{X}$ in $(\tilde{X}, \tilde{d}, E)$. Then $(F, E)$ is said to be soft open in $\tilde{X}$ with respect to $\tilde{d}$ if and only if all soft points of $(F, E)$ is interior points of $(F, E)$.

Definition 2.11. [7] Let $(\tilde{X}, \tilde{d}, E)$ and $\left(\tilde{Y}, \tilde{\rho}, E^{\prime}\right)$ be two soft metric spaces. The mapping $(f, \varphi):(\tilde{X}, \tilde{d}, E) \rightarrow\left(\tilde{Y}, \tilde{\rho}, E^{\prime}\right)$ is a soft mapping, where $f: X \rightarrow Y, \quad \varphi: E \rightarrow E^{\prime}$ are two mappings.

Definition 2.12. [8] Let $\left\{\tilde{x}_{\lambda, n}\right\}_{n}$ be a sequence of soft points in a soft metric space $(\tilde{X}, \tilde{d}, E)$. The sequence $\left\{\tilde{x}_{\lambda, n}\right\}_{n}$ is said to be convergent $(\tilde{X}, \tilde{d}, E)$ if there is a soft point $\tilde{y}_{\mu} \tilde{\in} \tilde{X}$ such that $\tilde{d}\left(\tilde{x}_{\lambda, n}, \tilde{y}_{\mu}\right) \rightarrow \overline{0}$ as $n \rightarrow \infty$.

This means for every $\tilde{\mathrm{O}} \tilde{>} \overline{0}$, chosen arbitrarily $\exists$ a natural $N=N(\tilde{\varepsilon})$ such that $\overline{0} \tilde{\leq} \tilde{d}\left(\tilde{x}_{\lambda, n}, \tilde{y}_{\mu}\right) \tilde{<} \tilde{\mathrm{o}}$, whenever $\mathrm{n}>N$.

Definition 2.13. [8] (Cauchy Sequence). A sequence $\left\{\tilde{x}_{\lambda, n}\right\}_{n}$ of soft points in $(\tilde{X}, \tilde{d}, E)$ is considered as a Cauchy sequence in $\tilde{X}$ if corresponding to every $\tilde{\mathrm{o}} \tilde{>} \overline{0}, \exists m \in \square$ such that $\tilde{d}\left(\tilde{x}_{\lambda, i}, \tilde{x}_{\lambda, j}\right) \tilde{\leq} \tilde{\mathrm{o}}, \forall i, j \geq m$, i.e. $\tilde{d}\left(\tilde{x}_{\lambda, i}, \tilde{x}_{\lambda, j}\right) \rightarrow \overline{0}$ as $i, j \rightarrow \infty$. 
International Journal on Cybernetics \& Informatics (IJCI) Vol. 4, No. 5, October 2015

Definition 2.14. [8] (Complete Metric Space). A soft metric space $(\tilde{X}, \tilde{d}, E)$ is called complete if every Cauchy Sequence in $\tilde{X}$ converges to some point of $\tilde{X}$. The soft metric space $(\tilde{X}, \tilde{d}, E)$ is called incomplete if it is not complete.

\section{SOFT COMPATIBLE MAPS:}

Definition 3.1. Let $(A, \psi),(B, \varphi):(\tilde{X}, \tilde{d}, E) \rightarrow(\tilde{X}, \tilde{d}, E)$ be soft mappings. $(A, \psi)$ and $(B, \varphi)$ are said to be soft compatible if

$$
\lim _{n \rightarrow \infty} \tilde{d}\left((A, \psi)(B, \varphi) \tilde{x}_{\lambda_{n}}^{n},(B, \varphi)(A, \psi) \tilde{x}_{\lambda_{n}}^{n}\right)=\overline{0}
$$

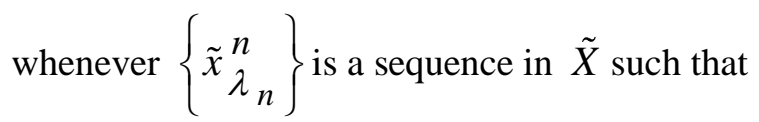

$$
\lim _{n \rightarrow \infty}(A, \psi) \tilde{x}_{\lambda_{n}}^{n}=\lim _{n \rightarrow \infty}(B, \varphi) \tilde{x}_{\lambda_{n}}^{n}=\tilde{z}_{v} \text { for some } \tilde{z}_{v} \text { in } \tilde{X}
$$

Definition 3.2. Let $(A, \psi),(B, \varphi):(\tilde{X}, \tilde{d}, E) \rightarrow(\tilde{X}, \tilde{d}, E)$ be soft mappings. $(A, \psi)$ and $(B, \varphi)$ are said to be soft compatible of type $(\alpha)$ if

$$
\lim _{n \rightarrow \infty} \tilde{d}\left((A, \psi)(B, \varphi) \tilde{x}_{\lambda_{n}}^{n},(B, \varphi)(B, \varphi) \tilde{x}_{\lambda_{n}}^{n}\right)=\overline{0}
$$

and

$$
\lim _{n \rightarrow \infty} \tilde{d}\left((B, \varphi)(A, \psi) \tilde{x}_{\lambda_{n}}^{n},(A, \psi)(A, \psi) \tilde{x}_{\lambda_{n}}^{n}\right)=\overline{0}
$$

whenever $\quad\left\{\begin{array}{c}\tilde{x}_{\lambda_{n}}^{n} \\ \lambda_{n}\end{array}\right\} \quad$ is $\quad$ a $\quad$ sequence $\quad$ in $\quad \tilde{X}$ such that $\lim _{n \rightarrow \infty}(A, \psi) \tilde{x}_{\lambda_{n}}^{n}=\lim _{n \rightarrow \infty}(B, \varphi) \tilde{x}_{\lambda_{n}}^{n}=\tilde{z}_{v}$ for some $\tilde{z}_{v}$ in $\tilde{X}$

Definition 3.3. Let $(A, \psi),(B, \varphi):(\tilde{X}, \tilde{d}, E) \rightarrow(\tilde{X}, \tilde{d}, E)$ be soft mappings. $(A, \psi)$ and $(B, \varphi)$ are said to be soft compatible of type $(\beta)$ if

$$
\lim _{n \rightarrow \infty} \tilde{d}\left((A, \psi)(A, \psi) \tilde{x}_{\lambda_{n}}^{n},(B, \varphi)(B, \varphi) \tilde{x}_{\lambda_{n}}^{n}\right)=\overline{0}
$$

whenever $\left\{\begin{array}{c}\tilde{x}_{\lambda_{n}}^{n} \\ \lambda\end{array}\right\}$ is a sequence in $\tilde{X}$ such that 
International Journal on Cybernetics \& Informatics (IJCI) Vol. 4, No. 5, October 2015

$\lim _{n \rightarrow \infty}(A, \psi) \tilde{x}_{\lambda_{n}}^{n}=\lim _{n \rightarrow \infty}(B, \varphi) \tilde{x}_{\lambda_{n}}^{n}=\tilde{z}_{v}$ for some $\tilde{z}_{v}$ in $\tilde{X}$

Definition 3.4. Let $(A, \psi),(B, \varphi):(\tilde{X}, \tilde{d}, E) \rightarrow(\tilde{X}, \tilde{d}, E)$ be soft mappings. $(A, \psi)$ and $(B, \varphi)$ are said to be $(\alpha)$-soft compatible mappings if

$$
\lim _{n \rightarrow \infty} \tilde{d}\left((A, \psi)(B, \varphi) \tilde{x}_{\lambda_{n}}^{n},(B, \varphi)(B, \varphi) \tilde{x}_{\lambda_{n}}^{n}\right)=\overline{0}
$$

whenever $\left\{\begin{array}{c}\tilde{x}_{\lambda_{n}}^{n} \\ \lambda_{n}\end{array}\right.$ is a sequence in $\tilde{X}$ such that

$$
\lim _{n \rightarrow \infty}(A, \psi) \tilde{x}_{\lambda_{n}}^{n}=\lim _{n \rightarrow \infty}(B, \varphi) \tilde{x}_{\lambda_{n}}^{n}=\tilde{z}_{v} \text { for some } \tilde{z}_{v} \text { in } \tilde{X}
$$

Definition 3.5. Let $(A, \psi),(B, \varphi):(\tilde{X}, \tilde{d}, E) \rightarrow(\tilde{X}, \tilde{d}, E)$ be soft mappings. $(A, \psi)$ and $(B, \varphi)$ are said to be $(\beta)$-soft compatible mappings if

$$
\lim _{n \rightarrow \infty} \tilde{d}\left((B, \varphi)(A, \psi) \tilde{x}_{\lambda_{n}}^{n},(A, \psi)(A, \psi) \tilde{x}_{\lambda_{n}}^{n}\right)=\overline{0}
$$

whenever $\left\{\begin{array}{c}\tilde{x}_{\lambda_{n}}^{n} \\ \lambda_{n}\end{array}\right.$ is a sequence in $\tilde{X}$ such that

$\lim _{n \rightarrow \infty}(A, \psi) \tilde{x}_{\lambda_{n}}^{n}=\lim _{n \rightarrow \infty}(B, \varphi) \tilde{x}_{\lambda_{n}}^{n}=\tilde{z}_{v}$ for some $\tilde{z}_{v}$ in $\tilde{X}$

Definition 3.6. Let $(A, \psi),(B, \varphi):(\tilde{X}, \tilde{d}, E) \rightarrow(\tilde{X}, \tilde{d}, E)$ be soft mappings. $(A, \psi)$ and $(B, \varphi)$ are said to be soft compatible of type $(I)$ if

$$
\varlimsup_{n \rightarrow \infty} \tilde{d}\left((A, \psi)(B, \varphi) \tilde{x}_{\lambda_{n}}^{n}, \tilde{z}_{v}\right) \tilde{z} \tilde{d}\left((B, \varphi) \tilde{z}_{v}, \tilde{z}_{v}\right)
$$

whenever $\left\{\begin{array}{c}\tilde{x}_{\lambda_{n}}^{n} \\ \lambda\end{array}\right.$ is a sequence in $\tilde{X}$ such that

$$
\lim _{n \rightarrow \infty}(A, \psi) \tilde{x}_{\lambda_{n}}^{n}=\lim _{n \rightarrow \infty}(B, \varphi) \tilde{x}_{\lambda_{n}}^{n}=\tilde{z}_{v} \text { for some } \tilde{z}_{v} \text { in } \tilde{X}
$$

Definition 3.7. Let $(A, \psi),(B, \varphi):(\tilde{X}, \tilde{d}, E) \rightarrow(\tilde{X}, \tilde{d}, E)$ be soft mappings. $(A, \psi)$ and $(B, \varphi)$ are said to be soft compatible of type $(I I)$ if

$$
\varlimsup_{n \rightarrow \infty} \tilde{d}\left((B, \varphi)(A, \psi) \tilde{x}_{\lambda_{n}}^{n}, \tilde{z}_{v}\right) \tilde{z} \tilde{d}\left((A, \psi) \tilde{z}_{v}, \tilde{z}_{v}\right)
$$


International Journal on Cybernetics \& Informatics (IJCI) Vol. 4, No. 5, October 2015

whenever $\left\{\begin{array}{c}\tilde{x}_{\lambda_{n}}^{n} \\ \lambda_{n}\end{array}\right.$ is a sequence in $\tilde{X}$ such that

$\lim _{n \rightarrow \infty}(A, \psi) \tilde{x}_{\lambda_{n}}^{n}=\lim _{n \rightarrow \infty}(B, \varphi) \tilde{x}_{\lambda_{n}}^{n}=\tilde{z}_{v}$ for some $\tilde{z}_{v}$ in $\tilde{X}$

Proposition 3.8. Let $(A, \psi),(B, \varphi):(\tilde{X}, \tilde{d}, E) \rightarrow(\tilde{X}, \tilde{d}, E)$ be soft mappings with $(B, \varphi)$ be soft continuous. Then $(A, \psi)$ and $(B, \varphi)$ are soft compatible if and only if they are $(\alpha)$ - soft compatible.

Proof. Suppose that $(A, \psi)$ and $(B, \varphi)$ are soft compatible and let $\left\{\tilde{x}_{\lambda_{n}}^{n}\right\}$ be a soft sequence in $\tilde{X}$ such that

$$
\lim _{n \rightarrow \infty}(A, \psi) \tilde{x}_{\lambda_{n}}^{n}=\lim _{n \rightarrow \infty}(B, \varphi) \tilde{x}_{\lambda_{n}}^{n}=\tilde{z}_{v}
$$

for some $\tilde{z}_{v}$ in $\tilde{X}$. By the triangle inequality, we have

$$
\begin{aligned}
& \tilde{d}\left((A, \psi)(B, \varphi) \tilde{x}_{\lambda_{n}}^{n},(B, \varphi)(B, \varphi) \tilde{x}_{\lambda_{n}}^{n}\right) \\
& \tilde{\leq} \tilde{d}\left((A, \psi)(B, \varphi) \tilde{x}_{\lambda_{n}}^{n},(B, \varphi)(A, \psi) \tilde{x}_{\lambda_{n}}^{n}\right)+ \\
& \tilde{d}\left((B, \varphi)(A, \psi) \tilde{x}_{\lambda_{n}}^{n},(B, \varphi)(B, \varphi) \tilde{x}_{\lambda_{n}}^{n}\right)
\end{aligned}
$$

it follows that

$$
\lim _{n \rightarrow \infty} \tilde{d}\left((A, \psi)(B, \varphi) \tilde{x}_{\lambda_{n}}^{n},(B, \varphi)(B, \varphi) \tilde{x}_{\lambda_{n}}^{n}\right)=\overline{0}
$$

Therefore, $(A, \psi)$ and $(B, \varphi)$ are $(\alpha)$ - soft compatible.

Conversely, suppose that $(A, \psi)$ and $(B, \varphi)$ are $(\alpha)$ - soft compatible. We then have

$$
\begin{aligned}
& \tilde{d}\left((A, \psi)(B, \varphi) \tilde{x}_{\lambda_{n}}^{n},(B, \varphi)(A, \psi) \tilde{x}_{\lambda_{n}}^{n}\right) \\
& \tilde{\leq} \tilde{d}\left((A, \psi)(B, \varphi) \tilde{x}_{\lambda_{n}}^{n},(B, \varphi)(B, \varphi) \tilde{x}_{\lambda_{n}}^{n}\right)+ \\
& \tilde{d}\left((B, \varphi)(B, \varphi) \tilde{x}_{\lambda_{n}}^{n},(B, \varphi)(A, \psi) \tilde{x}_{\lambda_{n}}^{n}\right)
\end{aligned}
$$

which implies that 
International Journal on Cybernetics \& Informatics (IJCI) Vol. 4, No. 5, October 2015

$$
\lim _{n \rightarrow \infty} \tilde{d}\left((A, \psi)(B, \varphi) \tilde{x}_{\lambda_{n}}^{n},(B, \varphi)(A, \psi) \tilde{x}_{\lambda_{n}}^{n}\right)=\overline{0}
$$

Therefore, $(A, \psi)$ and $(B, \varphi)$ are soft compatible. This completes the proof.

Proposition 3.9. Let $(A, \psi),(B, \varphi):(\tilde{X}, \tilde{d}, E) \rightarrow(\tilde{X}, \tilde{d}, E)$ be soft mappings with $(A, \psi)$ be soft continuous. Then $(A, \psi)$ and $(B, \varphi)$ are soft compatible if and only if they are $(\beta)$ - soft compatible.

Proof. The proof is obvious.

Proposition 3.10. Let $(A, \psi),(B, \varphi):(\tilde{X}, \tilde{d}, E) \rightarrow(\tilde{X}, \tilde{d}, E)$ be soft mappings such that $(B, \varphi)($ resp., $(A, \psi))$ be soft continuous maps. If $(A, \psi)$ and $(B, \varphi)$ are $(\alpha)$ soft compatible (resp., $(\beta))$ then they are soft compatible of type $(I)($ resp., $(I I))$.

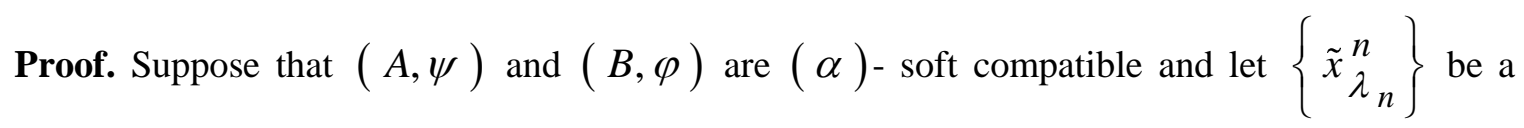
sequence in $\tilde{X}$ such that

$$
\lim _{n \rightarrow \infty}(A, \psi) \tilde{x}_{\lambda_{n}}^{n}=\lim _{n \rightarrow \infty}(B, \varphi) \tilde{x}_{\lambda_{n}}^{n}=\tilde{z}_{v}
$$

for some $\tilde{z}_{v}$ in $\tilde{X}$. Since $(B, \varphi)$ is soft continuous, we have

$$
\begin{aligned}
& \tilde{d}\left((A, \psi)(B, \varphi) \tilde{x}_{\lambda_{n}}^{n},(B, \varphi)(B, \varphi) \tilde{x}_{\lambda_{n}}^{n}\right)=\overline{0}=\tilde{d}\left((A, \psi)(B, \varphi) \tilde{x}_{\lambda_{n}}^{n},(B, \varphi) \tilde{z}_{v}\right) \\
& \text { and } \\
& \tilde{d}\left((B, \varphi) \tilde{z}_{v}, \tilde{z}_{v}\right) \tilde{\leq} \lim _{n \rightarrow \infty}\left(\tilde{d}\left((A, \psi)(B, \varphi) x_{\lambda_{n}}^{n}, z_{v}\right)+\right. \\
& \left.\tilde{d}\left((A, \psi)(B, \varphi) x_{\lambda_{n}}^{n},(B, \varphi) z_{v}\right)\right) \\
& =\lim _{n \rightarrow \infty}\left(\tilde{d}\left((A, \psi)(B, \varphi) \tilde{x}_{\lambda_{n}}^{n}, \tilde{z}_{v}\right)\right.
\end{aligned}
$$

Hence it follows that

$$
\varlimsup_{n \rightarrow \infty}\left(\tilde{d}\left((A, \psi)(B, \varphi) \tilde{x}_{\lambda_{n}}^{n}, \tilde{z}_{v}\right) \tilde{\geq} \tilde{d}\left((B, \varphi) \tilde{z}_{v}, \tilde{z}_{v}\right)\right.
$$


This inequality holds for every choice of the sequence $\left\{\tilde{x}_{\lambda_{n}}^{n}\right\}$ in $\tilde{X}$ with the corresponding $\tilde{z}_{v}$ in $\tilde{X}$ and so the $(A, \psi)$ and $(B, \varphi)$ are soft compatible of type $(I)$. This completes the proof.

Proposition 3.11. Let $(A, \psi),(B, \varphi):(\tilde{X}, \tilde{d}, E) \rightarrow(\tilde{X}, \tilde{d}, E)$ be soft mappings such that $(B, \varphi)($ resp., $(A, \psi))$ be soft continuous maps. If $(A, \psi)$ and $(B, \varphi)$ are soft compatible of type $(I)$ and for every sequence $\left\{\tilde{x}_{\lambda_{n}}^{n}\right\}$ in $\tilde{X}$ such that $\lim _{n \rightarrow \infty}(A, \psi) \tilde{x}_{\lambda_{n}}^{n}=\lim _{n \rightarrow \infty}(B, \varphi) \tilde{x}_{\lambda_{n}}^{n}=\tilde{z}_{v}$ for some $\tilde{z}_{v}$ in $\tilde{X}$, it follows that $\lim _{n \rightarrow \infty}(A, \psi)(B, \varphi) \tilde{x}_{\lambda_{n}}^{n}=\tilde{z}_{v}\left(\right.$ resp., $\left.\lim _{n \rightarrow \infty}(B, \varphi)(A, \psi) \tilde{x}_{\lambda_{n}}^{n}=\tilde{z}_{v}\right)$, then it is $(\alpha)$ - soft compatible (resp., $(\beta)$ - soft compatible ).

Proof. Suppose that $(A, \psi)$ and $(B, \varphi)$ are soft compatible of type $(I)$ and let $\left\{\begin{array}{c}\tilde{x}_{\lambda_{n}}^{n} \\ \lambda_{n}\end{array}\right.$ be a sequence in $\tilde{X}$ such that

$$
\lim _{n \rightarrow \infty}(A, \psi) \tilde{x}_{\lambda_{n}}^{n}=\lim _{n \rightarrow \infty}(B, \varphi) \tilde{x}_{\lambda_{n}}^{n}=\tilde{z}_{v} \text { for some } \tilde{z}_{v} \text { in } \tilde{X}
$$

Since $(B, \varphi)$ is soft continuous, we have

$$
\tilde{d}\left((B, \varphi) \tilde{z}_{v}, \tilde{z}_{v}\right) \tilde{\leq} \varlimsup_{n \rightarrow \infty}\left(\tilde{d}\left((A, \psi)(B, \varphi) \tilde{x}_{\lambda_{n}}^{n}, \tilde{z}_{v}\right)\right.
$$

and

$$
\begin{gathered}
\tilde{d}\left((B, \varphi)(B, \varphi) \tilde{x}_{\lambda_{n}}^{n}, \tilde{z}_{\nu}\right) \tilde{\leq} \varlimsup_{n \rightarrow \infty}\left(\tilde{d}\left((B, \varphi) \tilde{z}_{\nu}, \tilde{z}_{v}\right)+\right. \\
\left.\tilde{d}\left((B, \varphi)(B, \varphi) \tilde{x}_{\lambda_{n}}^{n},(B, \varphi) \tilde{z}_{v}\right)\right) \\
=\tilde{d}\left((B, \varphi) \tilde{z}_{v}, \tilde{z}_{v}\right)
\end{gathered}
$$

and so

$$
\lim _{n \rightarrow \infty} \tilde{d}\left((B, \varphi)(B, \varphi) \tilde{x}_{\lambda}^{n}, \tilde{z}_{v}\right) \tilde{\leq} \varlimsup_{n \rightarrow \infty}\left(\tilde{d}\left((A, \psi)(B, \varphi) \tilde{x}_{\lambda}^{n}, \tilde{z}_{v}\right)\right.
$$

Now we have 
International Journal on Cybernetics \& Informatics (IJCI) Vol. 4, No. 5, October 2015

$$
\begin{array}{r}
\tilde{d}\left((A, \psi)(B, \varphi) \tilde{x}_{\lambda_{n}}^{n},(B, \varphi)(B, \varphi) \tilde{x}_{\lambda_{n}}^{n}\right) \tilde{\leq} \tilde{d}\left((A, \psi)(B, \varphi) \tilde{x}_{\lambda_{n}}^{n}, \tilde{z}_{v}\right)+ \\
\tilde{d}\left((B, \varphi)(B, \varphi) \tilde{x}_{\lambda_{n}}^{n}, \tilde{z}_{v}\right)
\end{array}
$$

Hence, letting $n \rightarrow \infty$

$$
\begin{aligned}
& \varlimsup_{n \rightarrow \infty} \tilde{d}\left((A, \psi)(B, \varphi) \tilde{x}_{\lambda_{n}}^{n},(B, \varphi)(B, \varphi) \tilde{x}_{\lambda_{n}}^{n}\right) \\
& \tilde{\leq} \varlimsup_{n \rightarrow \infty} \tilde{d}\left((A, \psi)(B, \varphi) \tilde{x}_{\lambda_{n}}^{n}, \tilde{z}_{v}\right)+\varlimsup_{n \rightarrow \infty} \tilde{d}\left((B, \varphi)(B, \varphi) \tilde{x}_{\lambda_{n}}^{n}, \tilde{z}_{v}\right) \\
& \tilde{\leq} \varlimsup_{n \rightarrow \infty} \tilde{d}\left((A, \psi)(B, \varphi) \tilde{x}_{\lambda_{n}}^{n}, \tilde{z}_{v}\right)+\varlimsup_{n \rightarrow \infty} \tilde{d}\left((A, \psi)(B, \varphi) \tilde{x}_{\lambda_{n}}^{n}, \tilde{z}_{v}\right)
\end{aligned}
$$

Thus, $\lim _{n \rightarrow \infty} \tilde{d}\left((A, \psi)(B, \varphi) \tilde{x}_{\lambda_{n}}^{n},(B, \varphi)(B, \varphi) \tilde{x}_{\lambda_{n}}^{n}\right)=\overline{0}$.

Hence the $(A, \psi)$ and $(B, \varphi)$ are $(\alpha)$ - soft compatible. This completes the proof.

Proposition 3.12. Let $(A, \psi),(B, \varphi):(\tilde{X}, \tilde{d}, E) \rightarrow(\tilde{X}, \tilde{d}, E)$ be soft mappings such that $(B, \varphi)(\operatorname{resp} .,(A, \psi))$ be soft continuous maps. Suppose that, for every sequence $\left\{\begin{array}{c}\tilde{x}_{\lambda_{n}}^{n} \\ \lambda_{n}\end{array}\right\}$ in $\tilde{X}$ such that

$$
\lim _{n \rightarrow \infty}(A, \psi) \tilde{x}_{\lambda_{n}}^{n}=\lim _{n \rightarrow \infty}(B, \varphi) \tilde{x}_{\lambda_{n}}^{n}=\tilde{z}_{\nu} \text { for some } \tilde{z}_{v} \text { in } \tilde{X}
$$

it follows that, $\lim _{n \rightarrow \infty}(A, \psi)(B, \varphi) \tilde{x}_{\lambda_{n}}^{n}=\tilde{z}_{v}$ (resp., $\left.\lim _{n \rightarrow \infty}(B, \varphi)(A, \psi) \tilde{x}_{\lambda_{n}}^{n}=\tilde{z}_{v}\right)$, Then $(A, \psi)$ and $(B, \varphi)$ is soft compatible of type $(I)(\operatorname{resp.,of~type}(I I))$, if and only if it is $(\alpha)$ - soft compatible (resp., $(\beta)$ - soft compatible $)$.

Proof. The proof is obvious.

Proposition 3.13. Let $(A, \psi),(B, \varphi):(\tilde{X}, \tilde{d}, E) \rightarrow(\tilde{X}, \tilde{d}, E)$ be soft mappings. Suppose that the $(A, \psi)$ and $(B, \varphi)$ are soft compatible of $\operatorname{type}(I)(\operatorname{resp} ., o f \operatorname{type}(I I))(A, \psi) \tilde{z}_{v}=(B, \varphi) \tilde{z}_{v}$

for some $\tilde{z}_{v}$ in $\tilde{X}$ then, 
International Journal on Cybernetics \& Informatics (IJCI) Vol. 4, No. 5, October 2015

$$
\begin{gathered}
\tilde{d}\left((A, \psi) \tilde{z}_{\nu},(B, \varphi)(B, \varphi) \tilde{z}_{\nu}\right) \tilde{\leq} \tilde{d}\left((A, \psi) \tilde{z}_{\nu},(A, \psi)(B, \varphi) \tilde{z}_{\nu}\right) \\
\left(\operatorname{resp} ., \tilde{d}\left((B, \varphi) \tilde{z}_{\nu},(A, \psi)(A, \psi) \tilde{z}_{\nu}\right) \tilde{\leq} \tilde{d}\left((B, \varphi) \tilde{z}_{\nu},(B, \varphi)(A, \psi) \tilde{z}_{\nu}\right)\right)
\end{gathered}
$$

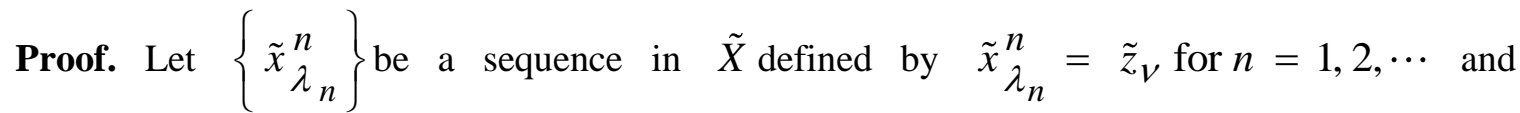
$(A, \psi) \tilde{z}_{v}=(B, \varphi) \tilde{z}_{v}$ for some $\tilde{z}_{v} \in \tilde{X}$. Then we have

$$
\lim _{n \rightarrow \infty}(A, \psi) \tilde{x}_{\lambda_{n}}^{n}=\lim _{n \rightarrow \infty}(B, \varphi) \tilde{x}_{\lambda_{n}}^{n}=\tilde{z}_{\nu}
$$

Suppose $(A, \psi)$ and $(B, \varphi)$ are soft compatible of type $(I)$. Then,

$$
\begin{gathered}
\tilde{d}\left((A, \psi) \tilde{z}_{v},(B, \varphi)(B, \varphi) \tilde{z}_{\nu}\right) \tilde{\leq} \tilde{d}\left((A, \psi) \tilde{z}_{\nu},(A, \psi)(B, \varphi) \tilde{x}_{\lambda_{n}}^{n}\right) \\
\tilde{\leq} \tilde{d}\left((A, \psi) \tilde{z}_{v},(A, \psi)(B, \varphi) \tilde{z}_{v}\right)
\end{gathered}
$$

Example 3.14. Let $(\tilde{X}, \tilde{d}, E)$ be a soft metric space with the following metrics

$$
\begin{gathered}
d(x, y)=|x-y|, d_{1}(x, y)=\min \{|x-y|, 1\}, \text { and } \\
\tilde{d}\left(x_{\lambda}, y_{\mu}\right)=\frac{1}{2}\left\{d_{1}(\lambda, \mu)+d(x, y)\right\}
\end{gathered}
$$

where $\quad \tilde{X}=[0, \infty)$ and $E=[0,1]$ Let function $A, B:[0, \infty) \rightarrow[0, \infty)$ and $\psi, \varphi:[0,1] \rightarrow[0,1]$ are defined as follows

$$
\begin{aligned}
& A \tilde{x}=\left\{\begin{array}{cl}
1 & \text { if } 0 \leq x \leq 1 \\
1+x & \text { if } 0<x<\infty
\end{array} \quad B \tilde{x}= \begin{cases}1+x & \text { if } 0 \leq x<1 \\
1 & \text { if } 1 \leq x<\infty\end{cases} \right. \\
& \psi(y)=\left\{\begin{array}{cc}
0 & \text { if } 0 \leq y \leq \frac{1}{2} \\
y & \text { if } \frac{1}{2}<y \leq 1
\end{array} \quad \varphi(y)=\left\{\begin{array}{cc}
y & \text { if } 0 \leq y<\frac{1}{2} \\
0 & \text { if } \frac{1}{2} \leq y \leq 1
\end{array}\right.\right.
\end{aligned}
$$

Let $\tilde{x}_{n}=y_{n}=\frac{1}{n}$

Then

$$
(A, \psi) \tilde{x}_{y_{n}}^{n}=(B, \varphi) \tilde{x}_{y_{n}}^{n}=(1)_{(0)}
$$

$(A, \psi)(B, \varphi) \tilde{x}_{y_{n}}^{n}=(A, \psi)\left(1+x_{n}\right) y_{n}=(1)(0)$

$(B, \varphi)(A, \psi) \tilde{x}_{y_{n}}^{n}=(B, \varphi)\left(1+x_{n}\right)_{\left(y_{n}\right)}=(2)_{(0)}$ 
International Journal on Cybernetics \& Informatics (IJCI) Vol. 4, No. 5, October 2015

$(A, \psi)(A, \psi) \tilde{x}_{y_{n}}^{n}=(A, \psi)\left(1+x_{n}\right)_{\left(y_{n}\right)}=(2)_{(0)}$

$(B, \varphi)(B, \varphi) \tilde{x}_{y_{n}}^{n}=(B, \varphi)\left(1+x_{n}\right) y_{n}=(2)(0)$

Consequently,

$$
\begin{aligned}
& \left.\lim _{n \rightarrow \infty} \tilde{d}\left((A, \psi)(B, \varphi) \tilde{x}_{\lambda_{n}}^{n},(B, \varphi)(B, \varphi) \tilde{x}_{\lambda_{n}}^{n}\right)\right) \neq \overline{0} \\
& \lim _{n \rightarrow \infty} \tilde{d}\left((A, \psi)(B, \varphi) \tilde{x}_{\lambda_{n}}^{n},(B, \varphi)(A, \psi) \tilde{x}_{\lambda_{n}}^{n}\right) \neq \overline{0}
\end{aligned}
$$

that is $(A, \psi)$ and $(B, \varphi)$ are neither soft compatible nor $(\alpha)$-soft compatible.

Example 3.15. Let $(\tilde{X}, \tilde{d}, E)$ be a soft metric space with the following metrics

$$
\begin{gathered}
d(x, y)=|x-y|, d_{1}(x, y)=\min \{|x-y|, 1\}, \text { and } \\
\tilde{d}\left(x_{\lambda}, y_{\mu}\right)=\frac{1}{2}\left\{d_{1}(\lambda, \mu)+d(x, y)\right\}
\end{gathered}
$$

where $\quad \tilde{X}=[0, \infty)$ and $E=[0,1]$ Let function $\quad A, B:[0, \infty) \rightarrow[0, \infty)$ and $\psi, \varphi:[0,1] \rightarrow[0,1]$ are defined as follows

$$
\begin{gathered}
A \tilde{x}=1 \quad \forall x \in \tilde{X} \quad B \tilde{x}= \begin{cases}1 & \forall x \neq 1 \\
2 & \forall x=1\end{cases} \\
\psi(y)=\left\{\begin{array}{ll}
\frac{1}{y^{3}} & \forall y \neq 0 \\
2 & \forall y=0
\end{array} \quad \varphi(y)= \begin{cases}\frac{1}{y^{2}} & \forall y \neq 0 \\
1 & \forall y=0\end{cases} \right.
\end{gathered}
$$

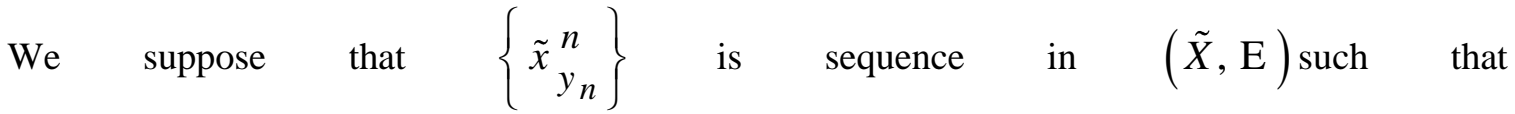
$\lim _{n \rightarrow \infty}(A, \psi)\left\{\tilde{x}_{y_{n}}^{n}\right\}=\lim _{n \rightarrow \infty}(B, \varphi)\left\{\begin{array}{c}\tilde{x}_{y_{n}}^{n} \\ y_{n}\end{array}\right\}=\tilde{z}_{v}$. By definition of

$$
(A, \psi) \text { and }(B, \varphi) \tilde{z}_{\nu} \in\{(1)(0)\}
$$

Since $A$ and $B$ agree on $\tilde{X},\{1\}$. We need only consider $\tilde{x}_{y_{n}}^{n} \rightarrow(1)$ ( 0 ). Now,

$$
\begin{gathered}
A B\left(x_{n}\right)=1, B A\left(x_{n}\right)=2, A A\left(x_{n}\right)=1, B B\left(x_{n}\right)=2, A(1)=1, \\
B(1)=2, \psi \varphi(y)=n^{6}, \varphi \psi(y)=n^{6}, \psi \psi(y)=n^{9} \\
\varphi \varphi(y)=n^{4}, \psi(0)=2, \varphi(0)=1
\end{gathered}
$$


Consequently,

$$
\begin{aligned}
& \lim _{n \rightarrow \infty} \tilde{d}\left((A, \psi)(B, \varphi) \tilde{x}_{\lambda_{n}}^{n},(B, \varphi)(B, \varphi) \tilde{x}_{\lambda_{n}}^{n}\right) \neq \overline{0} \\
& \lim _{n \rightarrow \infty} \tilde{d}\left((A, \psi)(B, \varphi) \tilde{x}_{\lambda_{n}}^{n},(B, \varphi)(A, \psi) \tilde{x}_{\lambda_{n}}^{n}\right) \neq \overline{0} \\
& \lim _{n \rightarrow \infty} \tilde{d}\left((A, \psi)(A, \psi) \tilde{x}_{\lambda_{n}}^{n},(B, \varphi)(A, \psi) \tilde{x}_{\lambda_{n}}^{n}\right) \neq \overline{0} \\
& \lim _{n \rightarrow \infty} \tilde{d}\left((A, \psi)(A, \psi) \tilde{x}_{\lambda_{n}}^{n},(B, \varphi)(B, \varphi) \tilde{x}_{\lambda_{n}}^{n}\right) \neq \overline{0}
\end{aligned}
$$

Therefore, $(A, \psi)$ and $(B, \varphi)$ are not soft compatible, soft compatible of type $(\alpha)$, soft compatible of type $(\beta),(\alpha)$ - soft compatible, $(\beta)$ - soft compatible.

$$
\lim _{n \rightarrow \infty} \tilde{d}\left((B, \varphi) 1_{0}, 1_{0}\right) \tilde{>} \frac{\overline{1}}{2}=\varlimsup_{n \rightarrow \infty} \tilde{d}\left((A, \psi)(B, \varphi) \tilde{x}_{\lambda_{n}}^{n}, 1_{0}\right)
$$

Therefore, the pair $((A, \psi)(B, \varphi))$ is not soft compatible of type $(I)$.

\section{FIXED POINT THEOREM:}

Theorem 4.1. Let $(\tilde{X}, \tilde{\mathrm{d}}, \mathrm{E})$ be a complete soft metric space and let $(A, \psi),(B, \varphi),(S, \phi)$ and $(T, \xi)$

be soft mappings from $\tilde{X}$ in to itself such that the following conditions are satisfied :

(a) $(A, \psi)(\tilde{X}, E) \subseteq(S, \phi)(\tilde{X}, E), \quad(B, \varphi)(\tilde{X}, E) \subseteq(T, \xi)(\tilde{X}, E)$

there exists a constant $\tilde{q} \in(\overline{0}, \overline{1})$ such that

$$
\begin{aligned}
d\left((A, \psi) \tilde{x}_{\lambda},(B, \varphi) \tilde{y}_{\mu}\right) \tilde{\leq} \tilde{q} \max \left\{d\left((S, \phi) \tilde{x}_{\lambda},(T, \xi) \tilde{y}_{\mu}\right),\right. \\
\tilde{d}\left((A, \psi) \tilde{x}_{\lambda},(S, \phi) \tilde{x}_{\lambda}\right), \tilde{d}\left((B, \varphi) \tilde{y}_{\mu},(T, \xi) \tilde{y}_{\mu}\right), \\
\left.\tilde{d}\left((A, \psi) \tilde{x}_{\lambda},(T, \xi) \tilde{y}_{\mu}\right), \tilde{d}\left((B, \varphi) \tilde{y}_{\mu},(S, \phi) \tilde{x}_{\lambda}\right)\right\}
\end{aligned}
$$

for all $\tilde{x}_{\lambda}, \tilde{y}_{\mu} \in \tilde{X}$. Let $(A, \psi),(B, \varphi),(S, \phi)$ and $(T, \xi)$ are satisfying conditions any one of the following :

(c) $\quad(A, \psi)$ is soft continuous and the pair $((A, \psi),(S, \phi))$ and $((B, \varphi),(T, \xi))$ are soft compatible of type ( II ).

(d) $(B, \varphi)$ is soft continuous and the pair $((A, \psi),(S, \phi))$ and $((B, \varphi),(T, \xi))$ are soft compatible of type ( II ).

(e) $\quad(S, \phi)$ is soft continuous and the pair $((A, \psi),(S, \phi))$ and $((B, \varphi),(T, \xi))$ 
International Journal on Cybernetics \& Informatics (IJCI) Vol. 4, No. 5, October 2015 are soft compatible of type (I ).

$(f) \quad(T, \xi)$ is soft continuous and the pair $((A, \psi),(S, \phi))$ and $((B, \varphi),(T, \xi))$ are soft compatible of type ( I ).

Then $(A, \psi),(B, \varphi),(S, \phi)$ and $(T, \xi)$ have a unique common fixed point in $\tilde{X}$.

Proof. Let $\tilde{x}_{\lambda_{0}}^{0} \in \operatorname{SP}(\tilde{X})$. From (a )we can construct sequence $\left\{\tilde{y}_{\mu_{n}}^{n}\right\}$ in $\operatorname{SP}(\tilde{X})$ such that

$$
(T, \xi) \tilde{x}_{\lambda_{2 n+1}}^{2 n+1}=(A, \psi) \tilde{x}_{\lambda_{2 n}}^{2 n}=\tilde{y}_{\mu_{2 n}}^{2 n}
$$

and

$$
(S, \phi) \tilde{x}_{\lambda_{2 n+2}}^{2 n+2}=(B, \varphi) \tilde{x}_{\lambda_{2 n+1}}^{2 n+1}=\tilde{y}_{\mu_{2 n+1}}^{2 n+1}
$$

we have

$$
\begin{aligned}
& \tilde{d}\left((A, \psi) \tilde{x}_{\lambda_{2 n}}^{2 n},(B, \varphi) \tilde{x}_{\lambda_{2 n+1}}^{2 n+1}\right) \tilde{\leq} q \max \left\{\tilde{d}\left((S, \phi) \tilde{x}_{\lambda_{2 n}}^{2 n},(T, \xi) \tilde{x}_{\lambda_{2 n+1}}^{2 n+1}\right),\right. \\
& \tilde{d}\left((A, \psi) \tilde{x}_{\lambda_{2 n}}^{2 n},(S, \phi) \tilde{x}_{\lambda_{2 n}}^{2 n}\right), \tilde{d}\left((B, \varphi) \tilde{x}_{\lambda_{2 n+1}}^{2 n+1},(T, \xi) \tilde{x}_{\lambda_{2 n+1}}^{2 n+1}\right), \\
& \left.\tilde{d}\left((A, \psi) \tilde{x}_{\lambda_{2 n}}^{2 n},(T, \xi) \tilde{x}_{\lambda_{2 n+1}}^{2 n+1}\right), \tilde{d}\left((B, \varphi) \tilde{x}_{\lambda_{2 n+1}}^{2 n+1},(S, \phi) \tilde{x}_{\lambda_{2 n}}^{2 n}\right)\right\}
\end{aligned}
$$

and so

$$
\begin{aligned}
& \tilde{d}\left(\tilde{y}_{\mu_{2 n}}^{2 n}, \tilde{y}_{\mu_{2 n+1}}^{2 n+1}\right) \tilde{\leq} q \max \left\{\tilde{d}\left(\tilde{y}_{\mu_{2 n-1}}^{2 n-1}, \tilde{y}_{\mu_{2 n}}^{2 n}\right), \tilde{d}\left(\tilde{y}_{\mu_{2 n}}^{2 n}, \tilde{y}_{\mu_{2 n-1}}^{2 n-1}\right), \tilde{d}\left(\tilde{y}_{\mu_{2 n+1}}^{2 n+1}, \tilde{y}_{\mu_{2 n}}^{2 n}\right),\right. \\
&\left.\tilde{d}\left(\tilde{y}_{\mu_{2 n}}^{2 n}, y_{\mu_{2 n}}^{2 n}\right), \tilde{d}\left(\tilde{y}_{\mu_{2 n+1}}^{2 n+1}, \tilde{y}_{\mu_{2 n-1}}^{2 n-1}\right)\right\} \\
& \tilde{\leq} q \max \left\{\tilde{d}\left(\tilde{y}_{\mu_{2 n-1}}^{2 n-1}, \tilde{y}_{\mu_{2 n}}^{2 n}\right), \tilde{d}\left(\tilde{y}_{\mu_{2 n}}^{2 n}, \tilde{y}_{\mu_{2 n+1}}^{2 n+1}\right)\right\}
\end{aligned}
$$

For $m, n \in N$, we have suppose $\mathrm{n}>\mathrm{m}$. Then we have

$$
\begin{aligned}
& \tilde{d}\left(\tilde{y}_{\mu_{n}}^{n}, \tilde{y}_{\mu_{m}}^{m}\right) \tilde{\leq} \tilde{d}\left(\tilde{y}_{\mu_{n}}^{n}, \tilde{y}_{\mu_{n+1}}^{n+1}\right)+\tilde{d}\left(\tilde{y}_{\mu_{n+1}}^{n+1}, \tilde{y}_{\mu_{n+2}}^{n+2}\right)+\cdots+ \\
& \tilde{d}\left(\tilde{y}_{\mu_{m-1}-1}^{m-\tilde{y}_{\mu_{m}}^{m}}\right) \\
& \\
& \tilde{\leq}\left(q^{m}+q^{m-1}+\cdots+q^{n-1}\right) \tilde{d}\left(\tilde{y}_{\mu_{0}}^{0}, \tilde{y}_{\mu_{1}}^{1}\right)
\end{aligned}
$$


International Journal on Cybernetics \& Informatics (IJCI) Vol. 4, No. 5, October 2015

$$
=\tilde{q}^{m} \frac{1-q^{(n-m)}}{1-\tilde{q}} \tilde{d}\left(\tilde{y}_{\mu_{0}}^{0}, \tilde{y}_{\mu_{1}}^{1}\right) \rightarrow \overline{0}(m \rightarrow \infty)
$$

and hence $\left\{\begin{array}{l}\tilde{y}_{\mu_{n}}^{n} \\ \mu_{n}\end{array}\right\}$ is a soft Cauchy sequence in $\tilde{X}$.

Since $(\tilde{X}, \tilde{\mathrm{d}}, \mathrm{E})$ is complete, $\left\{\begin{array}{c}\tilde{y}_{\mu_{n}}^{n} \\ \mu\end{array}\right\}$ converges to some soft point $\tilde{z}_{v} \in \tilde{X}$.

Also its subsequences converges to the same soft point i.e, $\tilde{z}_{v} \in \tilde{X}$.

Now, suppose that the condition (f) holds. Then, since the pair $((B, \varphi)(T, \xi))$ is soft compatible of type $(\mathrm{I})$ and $(T, \xi)$ is soft continuous, we have

$$
\begin{gathered}
\tilde{d}\left((T, \xi) \tilde{z}_{v}, \tilde{z}_{v}\right) \tilde{\leq} \varlimsup_{n \rightarrow \infty} \tilde{d}\left((B, \varphi)(T, \xi) \tilde{x}_{\lambda_{2 n+1}^{2 n+1}}^{2 n}, \tilde{z}_{v}\right) \\
(T, \xi)(T, \xi) \tilde{x}_{\lambda_{2 n+1}^{2 n+1}}^{2 n} \rightarrow(T, \xi) \tilde{z}_{v}
\end{gathered}
$$

Let $x_{\lambda}=\tilde{x}_{\mu_{2 n}}^{2 n}$ and $y_{\mu}=(T, \xi) \tilde{x}_{\lambda_{2 n+1}}^{2 n+1}$ in $(\mathrm{b})$, we obtain

$$
\tilde{d}\left((A, \psi) \tilde{x} \tilde{\lambda}_{2 n}^{2 n},(B, \varphi)(T, \xi) \tilde{x}_{\lambda_{2 n+1}}^{2 n+1}\right)
$$

$$
\left.\begin{array}{r}
\tilde{\leq} \tilde{q} \max \left\{\tilde{d}\left((S, \phi) \tilde{x}_{\lambda_{2 n}}^{2 n},(T, \xi)(T, \xi) \tilde{x}_{\lambda_{2 n+1}^{2 n+1}}^{2 n}\right), \tilde{d}\left((A, \psi) \tilde{x}_{\lambda_{2 n}}^{2 n},(S, \phi) \tilde{x}_{\lambda_{2 n}}^{2 n}\right.\right.
\end{array}\right),
$$

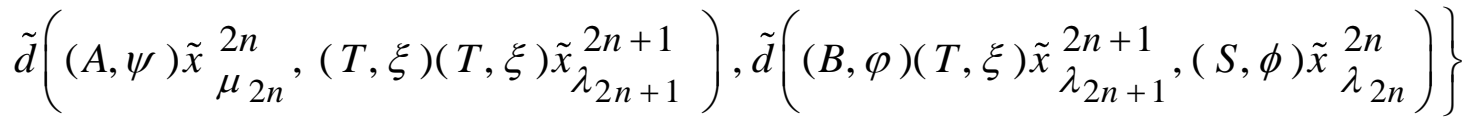

Thus, by letting the limit inferior on both sides of ( 1 ) we have

$$
\begin{aligned}
& \varlimsup_{n \rightarrow \infty} \tilde{d}\left(\tilde{z}_{V},(B, \varphi)(T, \xi) \tilde{x}_{\lambda_{2 n+1}}^{2 n+1}\right) \tilde{\leq} q \max \left\{\tilde{d}\left(\tilde{z}_{V},(T, \xi) \tilde{z}_{V}\right),\right. \\
& \left.\varlimsup_{n \rightarrow \infty} \tilde{d}\left((T, \xi) \tilde{z}_{v},(B, \varphi)(T, \xi) \tilde{x}_{\lambda_{2 n+1}^{2 n+1}}^{2 n}\right), \varlimsup_{n \rightarrow \infty} \tilde{d}\left(\tilde{z}_{v},(B, \varphi)(T, \xi) \tilde{x}_{\lambda_{2 n+1}^{2 n+1}}^{2 n}\right)\right\} \\
& \tilde{\leq} \tilde{q} \max \left\{\tilde{d}\left(\tilde{z}_{V},(T, \xi) \tilde{z}_{V}\right), \tilde{d}\left(\tilde{z}_{V},(T, \xi) \tilde{z}_{V}\right)+\varlimsup_{n \rightarrow \infty} \tilde{d}\left(\tilde{z}_{V},(B, \varphi)(T, \xi) \tilde{x}_{\lambda_{2 n+1}}^{2 n+1}\right),\right.
\end{aligned}
$$


International Journal on Cybernetics \& Informatics (IJCI) Vol. 4, No. 5, October 2015

$$
\begin{aligned}
& \left.\varlimsup_{n \rightarrow \infty} \tilde{d}\left(\tilde{z}_{V},(B, \varphi)(T, \xi) \tilde{x}_{\lambda_{2 n+1}}^{2 n+1}\right)\right\} \\
& \tilde{\leq} \tilde{q} \max \left\{\varlimsup_{n \rightarrow \infty} \tilde{d}\left(\tilde{z}_{v},(B, \varphi)(T, \xi) \tilde{x}_{\lambda_{2 n+1}^{2 n+1}}^{2 n}\right), \tilde{d}\left(\tilde{z}_{v},(B, \varphi)(T, \xi) \tilde{x}_{\lambda_{2 n+1}}^{2 n+1}\right)+\right. \\
& \left.\varlimsup_{n \rightarrow \infty} \tilde{d}\left(\tilde{z}_{\nu},(B, \varphi)(T, \xi) \tilde{x}_{\lambda_{2 n+1}^{2 n+1}}^{2 n}\right), \varlimsup_{n \rightarrow \infty} \tilde{d}\left(\tilde{z}_{\nu},(B, \varphi)(T, \xi) \tilde{x}_{\lambda_{2 n+1}}^{2 n+1}\right)\right\} \\
& \Rightarrow \varlimsup_{n \rightarrow \infty} \tilde{d}\left(\tilde{z}_{V},(B, \varphi)(T, \xi) \tilde{x}_{\lambda_{2 n+1}}^{2 n+1}\right) \tilde{\leq} \tilde{q} \varlimsup_{n \rightarrow \infty} \tilde{d}\left(\tilde{z}_{V},(B, \varphi)(T, \xi) \tilde{x} \begin{array}{c}
2 n+1 \\
\lambda_{2 n+1}
\end{array}\right) \\
& \Rightarrow \varlimsup_{n \rightarrow \infty} \tilde{d}\left(\tilde{z}_{V},(B, \varphi)(T, \xi) \tilde{x}_{\lambda_{2 n+1}}^{2 n+1}\right) \approx \varlimsup_{n \rightarrow \infty} \tilde{d}\left(\tilde{z}_{V},(B, \varphi)(T, \xi) \tilde{x}_{\lambda_{2 n+1}}^{2 n+1}\right)
\end{aligned}
$$

which is contradiction. Hence

$$
\varlimsup_{n \rightarrow \infty}(B, \varphi)(T, \xi) \tilde{x}_{\lambda_{2 n+1}}^{2 n+1}=\tilde{z}_{V}
$$

Now using the soft compatibility of type (I), we have

$$
\tilde{d}\left((T, \xi) \tilde{z}_{V}, \tilde{z}_{V}\right) \tilde{\leq} \varlimsup_{n \rightarrow \infty} \tilde{d}\left(\tilde{z}_{V},(B, \varphi)(T, \xi) \tilde{x}_{\lambda_{2 n+1}}^{2 n+1}\right)=\overline{0}
$$

and so it follows that $(T, \xi) \tilde{z}_{V}=\tilde{z}_{V}$.

Again, replacing $x_{\lambda}$ by $\tilde{x}_{\lambda_{2 n}}^{2 n}$ and $y_{\mu}$ by $\tilde{z}_{\nu}$ in $(b)$ we have

$$
\begin{aligned}
\tilde{d}\left((A, \psi) \tilde{x}_{\lambda_{2 n}}^{2 n},(B, \varphi) \tilde{z}_{v}\right) \tilde{\leq} \tilde{q} \max \left\{\tilde{d}\left((S, \phi) \tilde{x}_{\lambda_{2 n}}^{2 n},(T, \xi) \tilde{z}_{V}\right)\right. \\
\tilde{d}\left((A, \psi) \tilde{x}_{\lambda_{2 n}}^{2 n},(S, \phi) \tilde{x}_{\lambda_{2 n}}^{2 n}\right), \tilde{d}\left((B, \varphi) \tilde{z}_{V},(T, \xi) \tilde{z}_{v}\right) \\
\left.\tilde{d}\left((A, \psi) \tilde{x}_{\lambda_{2 n}}^{2 n},(T, \xi) \tilde{z}_{V}\right), \tilde{d}\left((B, \varphi) \tilde{z}_{V},(S, \phi) \tilde{x}_{\lambda_{2 n}}^{2 n}\right)\right\}
\end{aligned}
$$

and so, letting $n \rightarrow \infty$ we have

$\Rightarrow \tilde{d}\left((B, \varphi) \tilde{z}_{v}, \tilde{z}_{v}\right) \tilde{\leq} \tilde{q} \tilde{d}\left((B, \varphi) \tilde{z}_{v}, \tilde{z}_{v}\right) \tilde{<} \tilde{d}\left((B, \varphi) \tilde{z}_{v}, \tilde{z}_{v}\right)$

which is contradiction. Hence, $(B, \varphi) \tilde{z}_{V}=\tilde{z}_{V}$. Since,

$$
(B, \varphi)(\tilde{X}, E) \tilde{\subseteq}(T, \xi)(\tilde{X}, E)
$$

there exists a soft point $\tilde{u}_{\tilde{\mathrm{n}}} \in \tilde{X}$ such that

$(B, \varphi) \tilde{z}_{v}=(S, \phi) \tilde{u}_{\tilde{\mathrm{n}}}=\tilde{z}_{v}$ By $(\mathrm{b})$, we have 
International Journal on Cybernetics \& Informatics (IJCI) Vol. 4, No. 5, October 2015

$$
\begin{aligned}
& \tilde{d}\left((A, \psi) \tilde{u}_{\tilde{\mathrm{n}}}, \tilde{z}_{v}\right) \tilde{\leq} q \max \left\{\tilde{d}\left((S, \phi) \tilde{u}_{\tilde{\mathrm{n}}}, \tilde{z}_{v}\right), \tilde{d}\left((A, \psi) \tilde{u}_{\tilde{\mathrm{n}}},(S, \phi) \tilde{u}_{\tilde{\mathrm{n}}}\right),\right. \\
& \left.\tilde{d}\left(\tilde{z}_{v}, \tilde{z}_{v}\right), \tilde{d}\left((A, \psi) \tilde{u}_{\tilde{\mathrm{n}}}, \tilde{z}_{v}\right), \tilde{d}\left(\tilde{z}_{v},(S, \phi) \tilde{u}_{\tilde{\mathrm{n}}}\right)\right\}
\end{aligned}
$$

and so

$$
\tilde{d}\left((A, \psi) \tilde{u}_{\tilde{\mathrm{n}}}, \tilde{z}_{v}\right) \tilde{\leq} \tilde{q} \tilde{d}\left((A, \psi) \tilde{u}_{\tilde{\mathrm{n}}}, \tilde{z}_{v}\right) \tilde{<} \tilde{d}\left((A, \psi) \tilde{u}_{\tilde{\mathrm{n}}}, \tilde{z}_{v}\right)
$$

which is contradiction. Hence, $(A, \psi) \tilde{u}_{\tilde{\mathrm{n}}}=\tilde{z}_{v}$.

Since the pair $((A, \psi)(S, \phi))$ is soft compatible of type ( I ) and

$$
(A, \psi) \tilde{u}_{\tilde{\mathrm{n}}}=(S, \phi) \tilde{u}_{\tilde{\mathrm{n}}}=\tilde{z}_{v}
$$

By Proposition, we have

$$
\tilde{d}\left((A, \psi) \tilde{u}_{\tilde{\mathrm{n}}},(S, \phi)(S, \phi) \tilde{z}_{v}\right) \tilde{\leq} \tilde{q} \tilde{d}\left((A, \psi) \tilde{u}_{\tilde{\mathrm{n}}},(A, \psi)(S, \phi) \tilde{z}_{V}\right)
$$

and so

$$
\tilde{d}\left(\tilde{z}_{v},(S, \phi) \tilde{z}_{v}\right) \tilde{\leq} \tilde{q} \tilde{d}\left(\tilde{z}_{v},(A, \psi) \tilde{z}_{v}\right)
$$

Again, by ( $b$ ), we have

$$
\begin{gathered}
\tilde{d}\left((A, \psi) \tilde{z}_{v}, \tilde{z}_{v}\right) \tilde{\leq} \tilde{q} \max \left\{\tilde{d}\left((S, \phi) \tilde{z}_{v}, \tilde{z}_{v}\right), \tilde{d}\left((A, \psi) \tilde{z}_{V},(S, \phi) \tilde{z}_{v}\right), \tilde{d}\left(\tilde{z}_{V}, \tilde{z}_{v}\right),\right. \\
\left.\tilde{d}\left((A, \psi) \tilde{z}_{v}, \tilde{z}_{v}\right), \tilde{d}\left(\tilde{z}_{v},(S, \phi) \tilde{z}_{v}\right)\right\} \\
\tilde{\leq} \tilde{q} \tilde{d}\left((A, \psi) \tilde{z}_{v}, \tilde{z}_{v}\right) \tilde{<} \tilde{d}\left((A, \psi) \tilde{z}_{V}, \tilde{z}_{v}\right)
\end{gathered}
$$

which is contradiction. Hence, $(A, \psi) \tilde{z}_{v}=\tilde{z}_{v}$.

Therefore,

$$
(A, \psi) \tilde{z}_{V}=(B, \varphi) \tilde{z}_{V}=(S, \phi) \tilde{z}_{V}=(T, \xi) \tilde{z}_{V}=\tilde{z}_{V}
$$

and $\tilde{z}_{V}$ is a common fixed point of $(A, \psi),(B, \varphi),(S, \phi)$ and $(T, \xi)$.

The uniqueness of a common fixed point can be easily verified by using $(b)$.

The other cases ( c ), ( d ) and (e ) can be disposed from a similar argument as above.

\section{CONCLUSION}

In this paper, under different sufficient conditions several types of compatible maps are being compared in soft metric spaces and also fixed point theorem for four soft continuous self maps is established here. One can further try to generalize these concepts in fuzzy soft metric spaces, because, fuzzy soft metric space is a generalization of both fuzzy metric space and soft metric space. We hope that the findings in this paper will help researchers to enhance and promote the further study on soft metric spaces to carry out a general framework for their applications in real life.

\section{ACKNOWLEDGMENT}

Authors are thankful to the reviewer for his/her valuable comments. 


\section{REFERENCES}

[1] C. Gunduz (Aras), A. Sonmez, H. akall, On Soft Mappings , arXiv:1305.4545v1 [math.GM] 16 May 2013.

[2] D.Chen, The parameterization reduction of soft sets and its applications, Comput. Math. Appl. 49 (2005) $757-763$.

[3] D. Molodtsov, Soft set-theory-first results ,Comput. Math. Appl. 37 (1999)19-31

[4] M. Shabir and M. Naz, On soft topological spaces, Comput. Math. Appl.61 (2011) 1786- 1799.

[5] P. K. Maji, A.R.Roy, R. Biswas, An application of soft sets in a decision making problem, Comput. Math. Appl. 44 (2002) $1077-1083$

[6] P.K.Maji, R.Biswas, A.R.Roy, Soft set theory, Comput. Math. Appl. 45 (2003) $555-562$

[7] P. Majumdar, S.K. Samanta, On soft mappings, Comput. Math. Appl. 60 (2010) $2666-2672$.

[8] S. Das and S.K.Samanta, Soft metric, Annals of Fuzzy Mathematics and Informatics 6(1) (2013) 77 94

[9] S. Das and S.K.Samanta, Soft Real Sets, Soft Real Numbers and Their Properties, The journal of Fuzzy Mathematics 20(3) (2012) $551-576$

[10] S. Bayramov, C. Gunduz(Aras), Soft locally compact and soft paracompact spaces, Journal of Mathematics and System Science (accepted)

[11] S. Hussain and B. Ahmad, Some properties of soft topological spaces, Computers and Math with Applications 62 (2011) 4058 - 4067 .

[12] Sanjay Roy, T.K.Samanta, A note on Soft Topological Space, Punjab University Journal of mathematics, 46(1) (2014) $19-24$

[13] Sanjay Roy, T.K.Samanta, An introduction to open and closed sets on fuzzy soft topological spaces, Annals of Fuzzy mathematics and Informatics, 6(2) (2013) 425 - 431

[14] Sanjay Roy, T.K.Samanta, A note on fuzzy soft topological spaces, Annals of Fuzzy mathematics and Informatics, 3(2) (2012) $305-311$ 